\title{
Identificación de necesidades para proponer el desarrollo turístico en la comunidad de Tuncarta, del cantón Saraguro de la provincia de Loja
}

\section{Identification of needs to propose tourism development in the community of Tuncarta, of the Saraguro canton of the province of Loja}

Pablo Antonio Abrigo Córdova, Mgs.

Universidad Internacional del Ecuador, Ecuador

Autor para correspondencia: gavilondocarlos@gmail.com

Fecha de recepción: 28 de Junio de 2016 - Fecha de aceptación: 05 de Julio de 2016

Resumen: A lo alto de los Andes al sur del Ecuador, entre las provincias de Azuay y Loja, se ubica el cantón Saraguro. Ahí viven los Saraguros, una cultura indígena bien conocida por su bien preservada forma de vida, su ropa y artesanía tradicional, su lengua quichua y su gastronomía. El pueblo Saraguro mantiene orgulloso, los cohesivos culturales que han estructurado su vida por cientos de años. Han trabajado para preservar su identidad indígena desde la conquista española y continúan haciéndolo durante la modernización y globalización actual. Caminantes de todo el mundo van a experimentar la orgullosa comunidad de Saraguro en su pueblo nativo. Además de la vibrante y rica cultura, Saraguro también tiene gran belleza natural, flora, fauna y la oportunidad de muchas actividades de aventura. Casi toda la infraestructura de turismo es local, creando una experiencia de viaje auténtica y sostenible. Uno de los principales problemas que aquejan al cantón Saraguro y en particular la comunidad de Tuncarta, es la erosión de sus tierras, debido a la falta de vegetación, especialmente en las partes altas, producto de la deforestación e incendios forestales que ha sido objeto; así como la falta de concientización para el cuidado y preservación de los bosques primarios. Otro inconveniente identificado en la zona es la no existencia de un adecuado sistema de señalética turística y ambiental, aspecto que dificulta la apreciación y caracterización de las potencialidades del sector. Además desconocen los mecanismos para desarrollar un turismo sostenible en el lugar y el cuidado de sus recursos naturales. Tomando como referencia los antecedentes señalados, creemos que son elementos de juicio pertinentes para dar a conocer los mecanismos de identificación de malestares que hacen que la comunidad de Tuncarta no se desarrolle turísticamente (Rivero, 2010), para ello se utilizó en la investigación, la capacitación continua, aplicación de la MDP, grupos focales, grupos de discusión y seminarios dirigidos a los moradores de la comunidad, cuyos resultados fueron evidenciados a corto y largo plazo, promoviendo en los involucrados una conciencia plena de protección, cuidado ambiental y concienciación, así como también una mentalidad madura e integral desarrollada a través de trabajo asociativo y comunitario. Por otra parte, consideramos que la educación es un eje del buen vivir, porque, en el proceso educativo debe contemplar la preparación de los futuros ciudadanos y ciudadanas para una sociedad democrática, equitativa, inclusiva y pacífica. En tal virtud los beneficiados de éste proyecto ejercerán responsabilidades en el marco de la interculturalidad, del respeto a sus diversidades, y de la convivencia armónica y respetuosa con la naturaleza (Sumak Kawsay). (SENPLADES, 2013).

Palabras claves: desarrollo turístico; malestares; mdp 
Abstract: A high in the Andes in southern Ecuador, between the provinces of Azuay and Loja, lies the saraguro canton. Saraguros live there, look for an indigenous culture known for its wellpreserved lifestyle, clothing and traditional crafts, quichua language and cuisine. The people Saraguro proudly maintain the cohesive cultural factors that have structured their life for hundreds of years. They have worked to preserve their indigenous identity from the spanish conquest and continue during modernization and actual globalization. Worldwide walkers will experience the proud community of Saraguro in his native town. In addition to the vibrant and rich culture, Saraguro also has great natural beauty, flora, fauna and Opportunity of the many adventure activities. Almost the entire tourism infrastructure is local, creating an authentic travel experience and Sustainable. One of the main problems facing the canton Saraguro and in particular the community Tuncarta, is the erosion of their land, due to lack of vegetation, especially in the high contradictory product of deforestation and forest fires has been; and lack of awareness for the care and preservation of primary forests. Another drawback identified in the area is the absence of an adequate system of tourism and environmental signage, something that hinders appreciation and characterization of the potential of the sector. Furthermore unknown mechanisms to develop sustainable tourism in place and care of natural resources. Referencing the aforementioned background, we believe are relevant evidence to present identification mechanisms of ailments that make the community Tuncarta not touristically developed (Rivero, 2010), for it was used in the research, ongoing training, implementation of the MDP, focus groups, discussion groups and seminars for residents of the community, whose results were evidenced in the short and long term, promoting in involved full awareness of protection, environmental care and awareness, and as a mature and comprehensive mindset developed through partnership and community work. Moreover, we believe that education is an axis of good living, because, in the educational process should include the preparation of future citizens for a democratic, fair, inclusive and peaceful society. By virtue of the beneficiaries of this project responsibilities exercised within the framework of multiculturalism, respect for diversity, and harmonious and respectful coexistence with nature (Sumak Kawsay). (SENPLADES, 2013).

Key words: tourism development; discomforts; mdp

\section{Introducción}

Escaso desarrollo turístico en la comunidad de Tuncarta: El Proyecto de investigación es una compilación del programa de vinculación denominado "Programa de vinculación y desarrollo comunitario en la comunidad de Tuncarta", el cual se desarrolló en el sector de Tuncarta, Cantón Saraguro de la Provincia de Loja y nació a petición de la comunidad y como una alternativa de solución ante las principales problemáticas identificadas como la deforestación y la falta de capacitación para potencializar sus atractivos turísticos y culturales, así como la escasa concienciación ambiental, implicando de ésta manera, la participación de estudiantes de la Universidad Internacional del Ecuador Extensión Loja pertenecientes a la Carrera de Gestión Turística y Medio y estudiantes del Colegio Agropecuario "Ñukanchik Kawsay", cuyo beneficio fue para los habitantes del sector, ya que, ellos participaron activamente de este proceso capacitación continua, por tal razón, se logró fortalecer su cultura, tradiciones e identidad nacional. Esta investigación desde un inicio representó para la Carrera y la UIDE un fortalecimiento de su imagen por intermedio de los estudiantes al momento de realizar los aprendizajes. 
Además, otro factor importante a destacar es que no existen trabajos investigativos, sobre el desarrollo turístico en la comunidad de Tuncarta y de los pocos no existe difusión, lo que ha propiciado poca importancia en el sector, en toda la provincia de Loja y a nivel nacional; ya sea por el escaso conocimiento sobre metodologías que propicien la identificación de problemas.

Las actividades desplegadas en la presente investigación fortalecieron la relación directa entre los beneficios de la comunidad mediante la participación de los estudiantes y el buen vivir gestado en la comunidad.

La intervención de los estudiantes de la CGTMA mediante las capacitaciones y acciones directas en el entorno permitieron identificar de las potencialidades de los comuneros y sus malestares, garantizando la igualdad de oportunidades para los involucrados en este proceso.

Para definir los principales problemas que necesitan solución, los cuales son de real importancia en la investigación, fue necesario aplicar una matriz para el diagnóstico participativo (MDP) (Abrigo, 2012) a los moradores del sector de Tuncarta, la cual nos sirvió para determinar sus malestares y priorizarlos mediante la ejecución de proyectos. Además nos permitió conocer cuáles han sido las causas y los efectos que han generado este tipo de problemas. Esta actividad se la realizó mediante la aplicación de grupos focales, de discusión, entre otros.

En la presente investigación se propuso los siguientes objetivos:

Objetivo Primario:

- Elaborar un proyecto de autodesarrollo que permita la identificación de malestares en los habitantes del sector Tuncarta del cantón Saraguro.

Objetivo Secundario:

- Fundamentar teóricamente el desarrollo turístico mediante la identificación de contradicciones.

- Elaborar proyectos gestados con la comunidad mediante la aplicación de la MDP para proponer el desarrollo turístico en la comunidad de Tuncarta

Hipótesis: El desarrollo turístico ha disminuido sustancialmente, motivado por diferentes factores económicos, sociales y culturales en el sector de Tuncarta, lo cual puede ser mejorado si se identifican los problemas en el sector a través de la aplicación de la MDP desde el enfoque del autodesarrollo comunitario.

Los objetivos e hipótesis de la presente investigación están relacionados directamente, ya que se podrá proponer el desarrollo turístico en la comunidad de Tuncarta siempre y cuando se identifiquen sus malestares mediante la aplicación de la MDP, que concluyan con la ejecución de proyectos priorizados.

Explore la Importancia del problema: Para la identificación de malestares en los habitantes de la comunidad de Tuncarta, se propuso una fundamentación metodológica desde la perspectiva de la intervención comunitaria (Martínez, 2011) que consintió en reforestación de 
lugares aledaños, potenciar sus atractivos turísticos, concienciación ambiental, ejecutando y aplicando proyectos emblemáticos en la comunidad de Tuncarta.

El valor metodológico de la presente investigación, consistió en la aplicación de una sistemática de autodesarrollo comunitario que desarrolló los procesos de participación, cooperación conciencia crítica y la consecución definitiva de los proyectos gestados por la comunidad (Freire, 2010).

Además, el valor social, estuvo en los beneficios que representó para la comunidad la capacitación continua que recibieron por parte de los alumnos de la UIDE - Loja.

Por esta razón se impulsó un plan de reforestación de especies nativas del sector de Tuncarta a través de los estudiantes de la UIDE - Loja, el Colegio Agropecuario "Ñukanchik kawsay" y comunidad en general. El proyecto a lo largo de su realización obtuvo grades beneficios que fueron de mucha ayuda, ya que, permitió reforestar los linderos adyacentes a los terrenos de los comuneros cerca de 1500 especies.

Después se procedió a identificar los principales atractivos turísticos existentes y de esta manera desarrollar un turismo comunitario en el sector de Tuncarta, que a su vez no genere aspectos negativos o vaya contra la naturaleza que existe en el sector. Con esta información básica se dio inicio al mismo; esto quiere decir que este tipo de proyecto representa ser una actividad turística, cultural y natural. Se fue compartiendo conocimientos con los estudiantes del colegio y comunidad, con los cuales se llevó a cabo el proyecto, siendo necesario la identificando los atractivos del lugar sean éstos de aspecto cultural ósea las manifestaciones que caracterizaban al lugar, el tipo de gastronomía que se da en este lugar, siendo muy imprescindible destacar el tipo de cosecha que cada habitante realiza tiene que ver directamente con el tipo de comida única del lugar como por ejemplo la chicha de jora.

Para concluir se elaboraron rótulos que consistieron en la señalética de los principales atractivos de la zona, la cual fue colocada con la finalidad de identificar los aspectos culturales y naturales logrando que el turista consiga orientarse de mejor manera para llegar a la comunidad, representando para la comunidad y los moradores aumentar sus ingresos económicos y se sientan orgullosos de sus raíces.

En lo que respecta a la educación continua, se logró obtener buenos resultados, los mismos que ayudaron a adquirir nuevos conocimientos, mejorando el nivel académico y personal y en particular de la comunidad, y, con el desarrollo de las dinámicas activas y participativas se logró la integración de todos y también la satisfacción de que este tipo de actividades ayudara a potenciar el lugar por sus atractivos cultuales y naturales, su tradición, su gente, su identidad y tomar en cuenta que este tipo de diligencias mejorara la calidad vida de los habitantes en general.

Una de las características identificativas fue que todos los actores involucrados estaban satisfechos del trabajo en conjunto, de las actividades que con responsabilidad se llevaron a cabo y también de la actitud y mente positiva de trabajar y hacerlo sin recibir nada a cambio, se lo realizó de una manera desinteresada y se obtuvo buenos resultados. 
En síntesis, el propósito de esta investigación en el sector de Tuncarta, fue identificar sus principales problemas y desarrollar vínculos entre las instituciones implicadas en este proceso de regeneración de bosques primarios, cuyo resultado influirá de cierto modo en la obtención a largo plazo de un espacio digno para respirar. Además permitió dar valor a los principales atractivos turísticos mediante la aplicación de una señalética elaborada con materiales que son amigables con el medio.

Se logró sentar las bases para realizar una concientización ambiental para que el morador aplique una educación ambiental adecuada en el lugar.

Cabe destacar que el impacto generado por los proyectos en la comunidad de Tuncarta fue muy satisfactorio, ya que, permitió cuantificar y cualificar los resultados mediante el trabajo comunitario, medición de niveles de satisfacción por proyecto ejecutado, aplicación de test de conocimiento, implementación de señalética, siembra de especies, entre los más importantes.

Finalmente hay que considerar que la MDP fue muy útil por que permitió la identificación de problemas, los cuales se los puede resolver con el apoyo de los moradores de la comunidad representando ser nuevos proyectos.

\section{Métodos}

Se ejecutó una investigación empírica - acción - participación, valiéndonos para ello de la observación directa y la aplicación de entrevistas y la realización de talleres de reflexión, grupos focales y de discusión, mediante la capacitación en talleres y trabajo comunitario. Además se efectuaron muestreos de toda la zona, requiriendo de un equipo inter y multidisciplinario formado por gestores socioculturales intracomunitarios de varios integrantes de la comunidad y estudiantes de la UIDE. Fue necesario empezar esta propuesta con el conocimiento de las características agroecológicas sector en estudio.

Los métodos científicos que se utilizaron en la presente investigación fueron desglosados de la siguiente manera:

Análisis - Síntesis: Permitió analizar por partes los principales elementos del desarrollo comunitario turístico, así como la participación y cooperación. De esta manera se evidenció, como comprender y consolidar el tema; se dividió racionalmente las múltiples relaciones y componentes, a su vez se estableció la relación entre las partes previamente analizadas posibilitando el descubrimiento de relaciones y características generales entre los elementos de la realidad.

Inducción - Deducción: Para analizar la participación fue necesario partir del desarrollo comunitario en general y luego se analizó la misma en el contexto de la identificación de contradicciones en los moradores del sector en estudio. Con este método se procedió a orientar la investigación de lo particular a lo general y viceversa, permitiendo apreciar lo que existe de común en fenómenos individuales y aplicar a casos concretos los conocimientos de mayor generalidad. 
Histórico - Lógico: Se partió de la organización y análisis de la evolución histórica de los principales conceptos que aquí se tienen en cuenta (Desarrollo Comunitario, atractivos turísticos, especies silvestres, recursos naturales) para visualizar la trayectoria real de las causas y efectos que han generado el escaso desarrollo turístico en la comunidad de Tuncarta.

Las técnicas que se utilizaron en la presente investigación fueron desglosadas de la siguiente manera:

La observación: Visualizó la realidad del hecho a investigar.

La encuesta: Se la empleó para investigar masivamente determinados hechos o fenómenos que han propiciado el poco desarrollo turístico en la comunidad, así como los principales atractivos turísticos. El tipo de encuestas aplicadas fueron anónimas y las preguntas se desarrollaron de forma abierta, para lo cual se les solicitó ayuda para la contestación de las preguntas que se encuentran impresas en un cuestionario. Hay que considerar que al finalizar los proyectos se aplicaron encuestas para medir la satisfacción demostrada por la comunidad ante los trabajos culminados en los diferentes proyectos.

La entrevista a profundidad: Se la realizó a los representantes de organismos de apoyo social y a miembros de la comunidad de Tuncarta, a los cuales se los consideró como informantes claves, por ser hombres y mujeres de sabiduría y conocer las actividades del sector. Con las opiniones vertidas en la entrevista se elaboró una valoración sobre su situación, determinando sus potencialidades y debilidades que imposibilitan el proceso del desarrollo turístico.

La entrevista a profundidad a informantes claves permitió la libertad de expresión del entrevistado, valiéndose para ello de preguntas, cuya información sirvió para ampliar el trabajo investigativo. Este método permitió al interventor observar las conductas y la forma de sentir y de expresarse, para luego construir una valoración de éstos elementos (Marchioni, 1999).

Grupos focales: La aplicación de éste tipo de grupos constituyó favorablemente al cumplimiento metodológico y a los objetivos del trabajo investigativo enfocada en el principio de autodesarrollo, esta técnica fue aplicada a los representantes o grupos de la comunidad, los cuales están al tanto de sus dificultades y que afectan el desarrollo turístico del sector. Los grupos son: Tantanishpa Purina, Teresa de Calcuta, Rumiñahui, Kuri, Consejo Pastoral, Junta de Agua, Presidencia del Cabildo, Comité de Padres de Familia de la Escuela Lago de San Pablo y Consejo Académico del Colegio Agropecuario a Distancia "Ñukanchik Kawsay". La elección de este método se debe a que se evitan las relaciones entre los integrantes de un mismo grupo.

Las variables propuestas en el estudio son las siguientes:

Variables

Variable dependiente: Identificación de malestares en los habitantes de la comunidad de Tuncarta. 
Variable independiente: Desarrollo turístico en la comunidad de Tuncarta.

Método de subsecciones etiquedos (Descripcion de los participantes): Los participantes del proyecto pertenecen a la comunidad de Tuncarta (480 habitantes) del cantón Saraguro de la provincia de Loja. Las principales actividades están destinadas a la agricultura, ganadería con animales menores y pocos practican las artesanías en el sector.

Población, muestra y medición: Para la población y muestra se consideró a moradores del sector de Tuncarta. La muestra utilizada fue la probabilística estratificada, ya que, la selección se llevó a cabo en forma mecánica y objetiva, fueron procedimientos aleatorios en cada estrato; en este caso se investigó a moradores de la comunidad de Tuncarta.

Los niveles de medición de las variables fueron primeramente identificadas como cualitativas nominales; las cuales estuvieron representadas por categorías y, sus elementos se identificaron como cualidades o atributos que no se midieron numéricamente. En este caso las categorías de las variables no tuvieron orden jerárquico. Las encuestas y entrevistas facilitaron la recolección de la información, la cual fue suficiente para verificar los objetivos planteados y la hipótesis; las cuales fueron analizadas e interpretadas por la muestra seleccionada.

Diseño de la investigación: El diseño de la investigación fue cualitativa y cuantitativa. En la investigación cuantitativa sólo se reunió información que puedo ser medida mediante las especies sembradas y la colocación de señalética en la zona. Por otro lado, la investigación cualitativa se centró en la recopilación de información principalmente verbal en lugar de mediciones. Luego, la información obtenida fue analizada de una manera interpretativa, subjetiva, impresionista o incluso diagnóstica (Calero, 2000).

Características de los participantes: La participación de los moradores de la comunidad de Tuncarta fue fundamental para la realización de la investigación; ya que, sus conocimientos y sabiduría sirvió como referentes teóricos para completar la información; además fueron participes de las actividades de reforestación e implementación de señalética turística y ambiental en el lugar.

Matriz del diagnóstico participativo: Se la utiliza para identificar los principales malestares de la comunidad de Tuncarta y para darles solución. Es un conjunto de técnicas y herramientas que permite que las comunidades hagan su propio diagnóstico y de ahí comiencen a auto-gestionar su planificación y desarrollo. De esta manera, los participantes podrán compartir experiencias y analizar sus conocimientos, a fin de mejorar sus habilidades de planificación y acción. Aunque originariamente fue concebido para zonas rurales, muchas de las técnicas del DDP se pueden utilizar igualmente en comunidades urbanas (Verdejo, 2003).

\section{Resultados}

Fundamentación teórica del desarrollo turístico mediante la identificación de contradicciones: La realización de un análisis socio-económico fue muy importante para conocer la realidad en su entorno socio cultural, económico, ambiental y los recursos naturales que cuenta actualmente la comunidad de Tuncarta (Herrera \& Guzman, 2013); después se 
realizaron reuniones para identificar los principales problemas que necesitaron solución, para ello, fue necesario aplicar una matriz para el diagnóstico participativo, la cual nos sirvió para determinar sus malestares y priorizarlos. Además nos permitió conocer cuáles han sido las causas y los efectos que han generado este tipo de problemas. Esta actividad se la realizó mediante la aplicación de grupos focales. Es importante tomar en cuenta las características del sector en estudio, el cual pertenece a la parroquia Saraguro del cantón Saraguro y provincia de Loja; altitud $2850 \mathrm{msnm}$; con una temperatura media anual de $8^{\circ} \mathrm{C}$; precipitación anual media de $1100 \mathrm{~mm}$ y con una zona de vida bosque seco montano bajo (bs - MB), con vientos predominantes de Este a Oste, con una humedad relativa del 65 \%. (Abrigo, 2007).

A continuación presentamos la matriz utilizada.

Tabla 1. Matriz para el diagnóstico participativo, Tuncarta, octubre del 2012

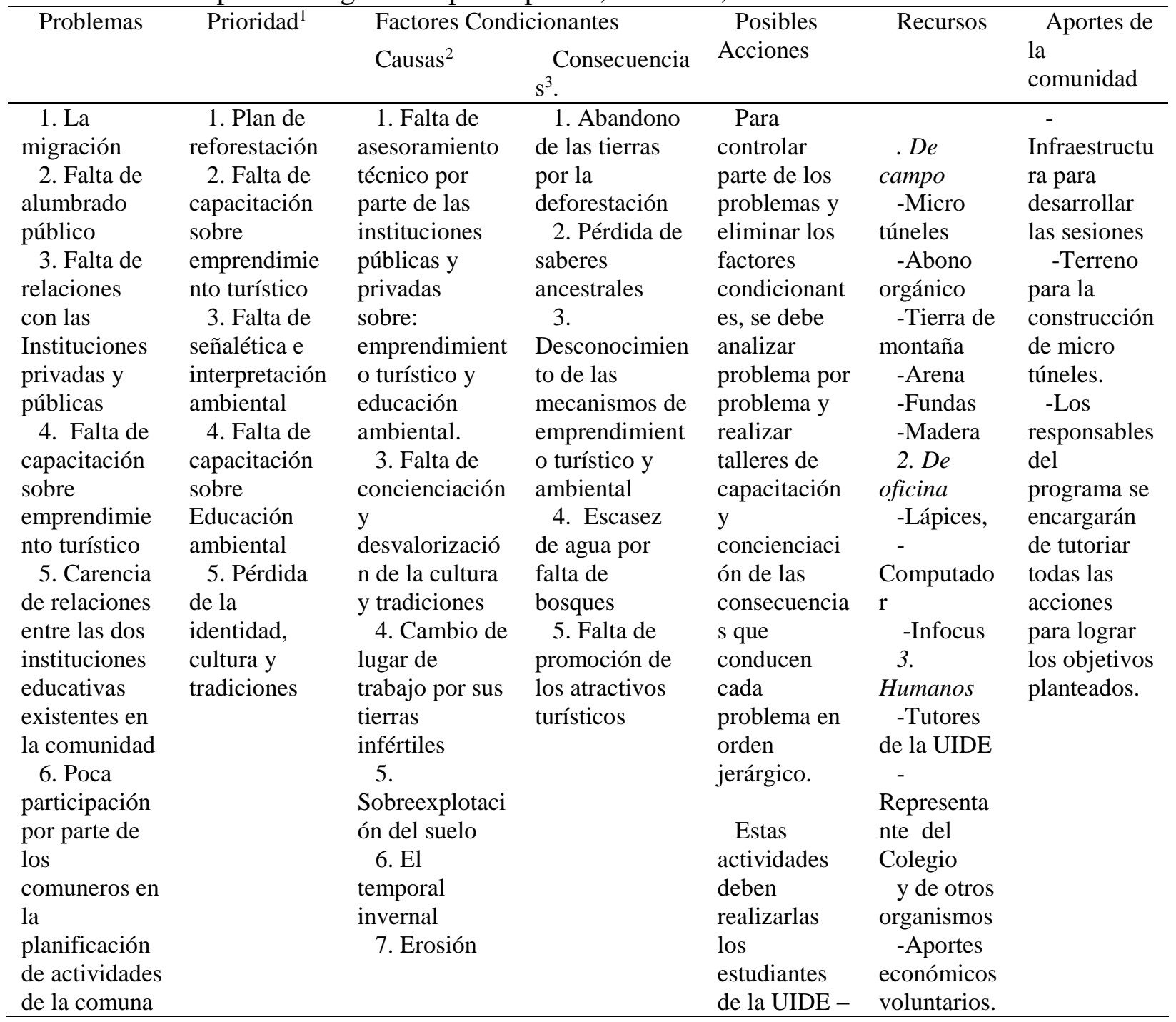

${ }^{1}$ Hay que considerar que, a los problemas se los priorizo para resolver las necesidades de los comuneros y para cumplir con los objetivos de la investigación; es por ello que no se utilizó ningún tipo de ponderación.

${ }^{2}$ Son causas que han incididos en los problemas detectados como prioritarios

${ }^{3}$ Son consecuencias derivadas de las causas 


\begin{tabular}{|c|c|c|}
\hline $\begin{array}{l}\text { 7. Vías de } \\
\text { acceso en } \\
\text { malas } \\
\text { condiciones } \\
\text { 8. No } \\
\text { existen } \\
\text { cooperativas } \\
\text { de producción } \\
\text { 9. Falta de } \\
\text { cooperación } \\
\text { por parte de } \\
\text { organismos } \\
\text { de desarrollo } \\
\text { 10. Falta de } \\
\text { capacitación } \\
\text { sobre } \\
\text { Educación } \\
\text { ambiental } \\
\text { 11. Pérdida } \\
\text { de la } \\
\text { identidad, } \\
\text { cultura y } \\
\text { tradiciones } \\
\text { 12. Pérdida } \\
\text { de los } \\
\text { cultivos } \\
\text { autóctonos } \\
\text { 13. Falta de } \\
\text { planes de } \\
\text { reforestación } \\
\text { 14. Falta de } \\
\text { señalética e } \\
\text { interpretación } \\
\text { ambiental }\end{array}$ & $\begin{array}{l}9 . \\
\text { Deforestación } \\
\text { masiva } \\
10 . \text { Falta de } \\
\text { conocimientos } \\
\text { sobre } \\
\text { valoración de } \\
\text { atractivos } \\
\text { turísticos y } \\
\text { señalética }\end{array}$ & $\begin{array}{l}\text { Loja bajo la } \\
\text { tutoría de los } \\
\text { docentes de } \\
\text { la UIDE }\end{array}$ \\
\hline
\end{tabular}

Proyectos gestados con la comunidad mediante la aplicación de la MDP para proponer el desarrollo turístico en la comunidad de Tuncarta: Los proyectos que propiciaron el desarrollo turístico de acuerdo a la aplicación de la matriz del diagnóstico participativo son: Plan de reforestación, Señalética e interpretación ambiental, y capacitación sobre educación ambiental, los cuales se detallan a continuación:

Plan de reforestación: En la comunidad de Tuncarta, perteneciente al cantón Saraguro, se desarrolló un proyecto de regeneración de la cubierta vegetal de las zonas afectadas por actividades de explotación maderera, agrícola y pastoril en la comunidad, para minimizar el impacto generado por las actividades humanas. Las acciones de reforestación se llevaron a cabo con la participación de estudiantes de la UIDE y el Colegio "Ñukanchik Kawsay", cuyo beneficio fue para los 480 habitantes del sector, cuyas actividades lograron fortalecer su cultura, tradiciones e identidad nacional. El Plan de reforestación propuesto, nació como resultado del estudio de la matriz para el diagnóstico participativo aplicado.

En el plan de reforestación se construyeron 13 micro túneles y un semillero que sirvieron para el establecimiento y manejo de especies nativas (sauce, mollon y aliso), las cuales fueron 
sembradas en los linderos de los comuneros. Hay que considerar que se propagaron cerca de 1500 plantas de especies nativas de las cuales se han sembrado el $80 \%$ en el sector y el $20 \%$ en comunidades aledañas de Namrin, Tambopamba, Gurudel entre otras.

Los procesos de transferencia a los beneficiarios se llevaron a cabo a través de capacitaciones, con la asistencia de estudiantes de las dos Instituciones involucradas y de personas de la comunidad, aplicándose un formato, donde se difundió los resultados que se obtuvieron durante la ejecución del proyecto de reforestación.

Hay que destacar que se aplicó un test de satisfacción a los comuneros por el trabajo realizado por los alumnos de la UIDE dando como resultados muy satisfactorios. También se firmaron actas de compromisos para el cuidado de las plantas por parte de los beneficiarios.

La finalidad de este proyecto fue: propiciar el sumak kawsay mediante los vínculos entre las instituciones implicadas en este proceso de regeneración de bosques primarios, cuyo resultado influirá positivamente en los habitantes de la comunidad.

Diseño de la interpretación y señalética turística - ambiental: En la actualidad no se da importancia por parte de los profesionales del área ambiental y turística para el reconocimiento de lugares turísticos e implementación de una señalética acorde al lugar. Es por ello que, resulta imprescindible para la Universidad Internacional del Ecuador Extensión Loja, apoyar a este tipo de proyectos, ya que, se pone de manifiesto la participación de sus alumnos, como gestores socioculturales (trasmisores de los conocimientos adquiridos en las aulas universitarias), con la comunidad que será beneficiada. Por este motivo se propuso una fundamentación metodológica desde la perspectiva de la intervención comunitaria enfocado en un diseño e interpretación turística en la comunidad de Tuncarta.

La finalidad de este proyecto fue desarrollar vínculos entre las instituciones implicadas en este proceso de trasmitir información técnica a información susceptible de aprender, además de facilitar a visitantes el recorrido por espacios seguros.

Antes de realizar las actividades planificadas en el proyecto se socializo con la comunidad la metodología a realizar en cada una de las etapas, para ello fue necesario vincular de manera directa a nuestros estudiantes y a los habitantes para lo cual se partió de las definiciones básicas para identificar los principales atractivos turísticos existentes y de esta manera desarrollar un turismo comunitario en el sector de Tuncarta, que a su vez no genere aspectos negativos o vaya contra la naturaleza que existe en el sector.

Con esta información básica se dio inicio al mismo; esto quiere decir que este tipo de proyecto representa ser una actividad turística, cultural y natural. Se fue compartiendo conocimientos con los estudiantes del colegio y comunidad, con los cuales se llevó a cabo el proyecto, siendo necesario la identificación de los atractivos del lugar sean éstos de aspecto cultural, ósea las manifestaciones que caracterizaban al lugar y el tipo de gastronomía (Ricaurte, 2009) siendo muy imprescindible destacar el tipo de cosecha que cada habitante realiza tiene que ver directamente con el tipo de comida única del lugar como por ejemplo la chicha de jora. 
Podemos mencionar que el arte culinario de esta comunidad es empírico, ya que, se transmite de generación en generación, perdurando de esta manera el folklore y las costumbres de esta zona de nuestra provincia.

Podemos encontrar platos típicos de sencilla elaboración que van desde una colada de zambo a otros más elaborados; cabe mencionar que toda la materia prima con la que elaboran la comida típica es sembrada y cosecha da de forma totalmente orgánica.

Otra manifestación que se da en él es la fabricación de sombreros que destacan al sector como productor de mismo, no solo en ese aspecto cultural sino también natural, ya que viven alrededor de pequeñas montañas donde se pueden realizar actividades de caminatas pero sin antes incluir un buen sendero. Todas estas actividades identificadas fueron reconocidas por los jóvenes que facilitaron la información para levantar las fichas, las cuales ayudaron a elaborar un inventario de atractivos del lugar.

Después de socializar con la comunidad y haber levantado un inventario de atractivos del lugar, se empezó a identificar los atractivos turísticos de la zona. Después de haber socializado esta actividad se llevó acabo el diseño de la ruta con ello se fue creando por medio de un croquis estableciendo los atractivos con ello contamos con aportaciones de los estudiantes fueron opinado. Luego del diseño se obtuvo la capacidad de carga de los senderos que tienen acceso hacia los atractivos identificados. Así como la elaboración de paquetes turísticos para el conocimiento de los turistas.

Para concluir con el proyecto se elaboraron rótulos que consistieron en la señalética de los principales atractivos de la zona, la cual fue colocada con la finalidad de identificar los aspectos culturales y naturales logrando que el turista logré orientarse de mejor manera para llegar a la comunidad, representando de esta manera para la comunidad y los moradores aumentar sus ingresos económicos y se sientan orgullosos de sus raíces.

Propuesta para la concienciación ambiental: El proyecto de educación ambiental se justifica a raíz de los problemas principales detectados a través de intervención realizada mediante los grupos focales, reflexión, entrevistas a profundidad y encuestas. Se ha observado una falta de concienciación, participación, motivación y cooperación por parte de toda la comunidad de Tuncarta, especialmente de los jóvenes. Se realizó una serie de actividades que permitieron de alguna manera, lograr la conciencia crítica y la transformación en su modo de pensar, actuar y vivir, para generar interés por preservar, conservar y cuidar el medio ambiente; lo cual lo logramos mediante la participación de toda la comunidad, hay que destacar que se realizaron prácticas de manejo de residuos generado por los habitantes, aplicando adecuadamente las 3 eres, e identificaron los principales recursos ambientales existentes en la comunidad.

Los impactos positivos fueron muy relevantes en el cambio de comportamiento hacia una mejor conciencia ambiental por parte de los pobladores de Tuncarta; es decir, los trabajos realizados e implementados han sido ejecutados tomando en cuenta las parámetros de sustentabilidad, o sea, cuando trabajamos en minga, rescatábamos el manejo ancestral, sin utilizar componentes no amigables al ambiente. 
No se ha identificado impactos negativos, ya que el programa dejo una conciencia ambiental más amigable con el torno.

Según las encuestas aplicadas a los beneficiarios se ha determinado que en un principio los conocimientos eran bajos (promedio de 6.7 equivalente a un $67 \%$ sobre 100), esto debido a que en la recopilación de campo se observó que existe cierta desinformación con respecto al medio ambiente; en comparación a los resultados obtenidos al finalizar el proyecto, estos fueron muy satisfactorios para la comunidad.

En lo que respecta al intercambio de conocimientos, se pudo evaluar, aplicando un segundo examen, cuyo promedio final obtenido fue de 18 sobre 20 , lo que evidencia que el intercambio realizado por la Academia a la colectividad fue muy satisfactorio.

\section{Discusión}

El enfoque aplicado en la investigación fue el mixto; es decir, se refiere a la metodología cualitativa que se empleó, representando al tamaño muestral necesario en función de encuestas, observaciones y entrevistas aplicadas; las cuales describieron la cotidianidad, aspectos socioculturales, situaciones problemáticas y los significados de la vida de las personas de la comunidad de Tuncarta.

La otra metodología que se utilizó fue, la cuantitativa, la cual se fundamentó en la búsqueda de las causas que generaron el poco desarrollo turístico, persiguiendo la razón y la explicación; permitiéndose dar cuenta de contextos, estructuras y alcance social de problemáticas que fueron tratadas en lo particular.

La utilización de estas dos metodologías permitió mayor profundización en este estudio, ya que, penetró en la subjetividad de los implicados, los motivó el tema a debatir y los hizo mantener una actitud más crítica de su situación actual. Además reflexionaron en torno a los mecanismos para superar la actual problemática y proyectarse a optimizar la calidad de vida y por ende un mejor porvenir.

Se utilizó la metodología del autodesarrollo para llevar a cabo el proceso de intervención comunitaria, en donde los resultados sirvieron para gestar el proyecto a identificar los malestares de los habitantes y proponer el desarrollo turístico.

Todos los comentarios expuestos por los asistentes a los eventos pusieron en evidencia los problemas de falta de participación por parte de los comuneros y cooperación por parte de los organismos de desarrollo, ya sean gubernamentales y no gubernamentales, en lo que tiene que ver con el ámbito turístico lo que consecuentemente propician el poco desarrollo.

Esta propuesta es la consecuencia de la gestación de lo comunitario, que se consiguió a través de la aplicación de la Matriz del Diagnósticio Participativo, donde se logró la identificación de los problemas y sus causas, el diagnóstico sobre el estado actual del turismo en el sector; así como la construcción colectiva de las acciones a emprender en la comunidad. 
La comunidad de Tuncarta debe establecer políticas y estrategias de gestión en el mantenimiento tanto de la señalética como de senderos, por medio de Juntas Parroquiales, Municipios, Empresa Privada y la colectividad. La sostenibilidad queda garantizada luego de la terminación del proyecto, ya que, la comunidad mantiene una organización bien estructurada, y además el compromiso y la apropiación del proyecto complementado todo esto con la facilitación de los conocimientos en cuanto al manejo y mantención de la infraestructura.

En el contexto social el proyecto aspira a ser uno de los medios más eficaces para generar la participación ciudadana dentro de la actividad turística, impulsando la creación de empleo y el desarrollo económico en la comunidad local.

También, se busca promover el respeto al medio ambiente por medio de la interpretación ambiental, acción que procura contribuir la conservación de los ecosistemas y la concientización de los visitantes.

Esta propuesta está dirigida a todos los segmentos de la población incluyendo la tercera edad y personas con capacidades especiales. En términos de género la recreación no es excluyente, es un vínculo para la cohesión social, familiar y laboral.

La Comunidad en mención, al no disponer de un inventario de atractivos turísticos elaborado, no cuenta con la herramienta básica para que la comunidad pueda despegar en forma definitiva, sustentable y sostenible en el ámbito turístico.

A través de este proyecto, el cual ha sido ejecutado por los estudiantes de la Carrera de Ingeniería en Gestión Turística y Medio Ambiente conjuntamente con alumnos del Colegio de Bachillerato "Nukanchik Kawsay" para Personas con Escolaridad Inconclusa perteneciente a la misma comunidad, cuyo beneficio fue para los 480 habitantes del sector, las actividades lograron fortalecer su cultura, tradiciones e identidad nacional. El Diseño propuesto, nació como resultado del estudio de la matriz para el diagnóstico participativo aplicado.

Este estudio se lo realizó mediante una revisión bibliográfica y con la ayuda de trabajos anteriores elaborados en la comunidad, así como testimonios de personas estudiosas y conocedoras del tema, se realizó la recolección de datos y elaboración de fichas, estudio, análisis y reflexión de la realidad turística de Tuncarta.

El impacto del proyecto en la comunidad ha sido muy relevante, ya que permitió la elaboración del inventario turístico en el que se han descrito los atractivos en una forma técnica, resaltando sus características, calidad, apoyo y significado, de acuerdo al manual del Ministerio de Turismo existente, se dispone del instrumento fundamental para emprender en nuevos proyectos dirigidos a potenciar y revitalizar el turismo comunal que sea más integrador. Se consideran dos paquetes turísticos los cuales se los difundió para potencializar turísticamente la comunidad de Tuncarta. Además se instaló señalética en el lugar, evidenciando los atractivos más representativos. La transmisión de las potencialidades identificadas técnicamente en el estudio a nivel nacional e internacional corresponderá a las autoridades de la comunidad y todos los involucrados en el turismo. 


\section{Conclusiones}

Los elementos epistemológicos observados en el transcurso de la investigación y en el proceso de intervención comunitaria fueron: temor al expresarse los estudiantes y moradores, miedo por parte del interventor a que salgan mal los talleres.

Se logró la conciencia crítica en los moradores del sector sobre los problemas que tiene la comunidad llegando a reflexionar al momento de solucionarlos.

El nivel de satisfacción de los comuneros por la calidad del trabajo realizado en los proyectos fue muy satisfactorios.

La Comunidad de Tuncarta dispone de los diferentes atractivos turísticos, como sitios naturales y manifestaciones culturales

El turismo en la Comunidad se ha constituido en una actividad que generaría ingresos económicos a los diferentes sectores.

Incrementar acciones de buenas prácticas de turismo sostenible en las comunidades

Se logró proponer el desarrollo turístico mediante la ejecución de los proyectos.

\section{Agradecimientos}

Nuestro agradecimiento imperecedero a los docentes y alumnos de la Carrera en Gestión Turística y Medio Ambiente de la Universidad Internacional del Ecuador extensión Loja y del Colegio Agropecuario NukanchikKawsay (Nuestra Vida), quienes con su sacrificio y entrega mostrado cada semana permitieron que este trabajo llegué a su feliz término.

De nosotros, especial testimonio de reconocimiento a la comunidad de Tuncarta, especialmente a los Directivos del Colegio, por la colaboración al brindarnos las facilidades para el desarrollo de la investigación en mención y a todos que de alguna manera impulsaron para que se tenga el éxito deseado.

\section{Bibliografía}

Abrigo, P., (2007). Producción y procesamiento para la comercialización de tres ecotipos de melloco en Saraguro. Tésis Ing. Agr. Loja, Ec., Universidad Nacional de Loja. Área Agropecuaria y de Recursos Naturales Renovables.

Abrigo, P., (2012). Los cultivos autóctonos en el sector de tuncarta del cantón saraguro y su perspectiva de rescate como aporte al desarrollo comunitario. Tésis Mgs. Loja, Ec., Universidad Nacional de Loja. Área Jurídica, Social y Administrativa.

Calero J. (2000). Investigación cualitativa y cuantitativa. Problemas no resueltos en los debates actuales. Rev. Cubana Endocrinol; 11 (3): 192-8. 
Freire, J. (2010). La investigación como herramienta para el desarrollo comunitario. Universidad Central "Martha Abrew" de la Villas, Santa Clara - Cuba.

Herrera, L. N., \& Guzman, P. V. (2013). Plan de desarrollo local para la comunidad Vicente León, parroquia Toacaso, cantón Latacunga, provincia de Cotopaxi. Tésis Eco. Latacunga, Ec., Universidad Central del Ecuador, Facultad de Ciencias Económicas.

Marchioni, M., (1999). Comunidad, participación y desarrollo. Madrid., Popular S.A.

Martínez, M. (2011). Desarrollo Sociocultural comunitario. Universidad Central "Martha Abrew" de la Villas, Santa Clara - Cuba.

Ricaurte Quijano, C. V. (2009). Manual para el diagnóstico turístico local.

Rivero, R., (2010). Intervención profesional en trabajo social y comunitario. Universidad Central "Martha Abrew" de la Villas, Santa Clara - Cuba.

Secretaria Nacional de Planificación y Desarrollo. (2013)., Plan Nacional delñ Buien vivir 2013 - 2017. Quito - Ecuador.

Verdejo, M. E., \& Hidalgo, E. J. (2003). Diagnóstico rural participativo: Una guía práctica. Centro Cultural Poveda, Proyecto Comunicación y Didáctica. 